\title{
Experimentelle Untersuchungen über das Staphylokokkengeschwür der Hornhaut und dessen Therapie.
}

\author{
Von \\ Dr. med. Ludwig Bach, \\ Privatdocenten und 1. Assistenzarzt der Universitäts-Augen-Klinik \\ zu Würzburg.
}

In der letzten Zeit wurden subconjunctivale Sublimatinjectionen von verschiedenen Seiten, besonders von Darier in Paris, empfohlen und zwar einmal gegen infectiöse Processe des Auges, weiterhin gegen alle möglichen Erkrankungen des Sehorganes, welche mit Bestimmtheit oder mehr oder minder grosser Wahrscheinlichkeit auf Lues acquisita oder congenita zurückzuführen sind.

Da mir aus verschiedenen Gründen eine therapeutische Wirkung derartiger Sublimatinjektionen sehr zweifelhaft erschien, beschloss ich auf experimentellem Wege der Prüfung dieser Frage näher zu treten.

Versuchsanordnung: Erzeugung eines Geschwüres auf beiden Hornhäuten eines Kaninchens durch Bildung einer oberflächlich gelegenen Tasche mittels der krummen Lanze und Einimpfung einer Reincultur von Staphylococcus pyogenes aureus mittels der krummen Lanze oder Platinspatels. Bei meinen Versuchen iiber den Werth der subconjunctivalen Sublimatinjectionen benutzte ich ausschliesslich Kaninchen. 
Experimentelle Unters. üb. d. Staphylokokkengeschwür etc. 57

Bemerkung zu diesem Infectionsmodus: Es gelingt nicht, bei anscheinend gleicher Einimpfung und Benutzung derselben Staphylokokkenreincultur immer den gleichen Intensitätsgrad der Geschwürsbildung hervorzurufen. Daraus folgt, dass es nicht angängig ist, aus dem Intensitätsgrad des auf genannte Weise erzengten Geschwüres direct Rückschlüsse zu machen auf die Virulenz des benutzten Impfmaterials. Die Heranziehung besonders kräftiger Kaninchen und andererseits von schwächlichen kleinen Thieren liess im allgemeinen nicht die Ueberzeugung aufkommen, dass erstere resp. deren Hornhaut widerstandsfähiger seien.

Was die Stelle der Hornhaut anlangt, an welcher ich die Implantation des Impfmaterials vornahm, so wählte ich die centralen Parthien der Hornhaut zur Anlegung des Geschwires, da es bei der Wahl dieser Stelle ausnahmslos gelingt, ein Geschwür der Hornhaut mit secundärer Entzündung der Iris, des Corpus ciliare und des vorderen Theils der Aderhaut zu erzeugen. Zunächst bildet sich fast immer an der Stelle der Infection eine rundliche, gelblich-weiss gefärbte Erhebung, erst dann ein sogenanntes offenes Geschwür. Es ist in dieser Hinsicht gleichgültig, ob man zur Erzeugung des Geschwürs eine Tasche gebildet oder in einen Oberflächenverlust der Hornhaut die Staphylokokken eingerieben hat. Die weiteren Veränderungen, die sich an und un die Implantationsstelle abspielen, erwähne ich hier nicht weiter, da dieselben von mehreren Forschem, ich nenne hier nur Leber, Michel, Rindfleisch, Hess, Silvestri, genau beschrieben sind und meine Beobachtungen sich damit decken.

Ich sagte oben, dass ich als Ort der Implantation die mittleren Parthien der Hornhaut wählte. Dies hatte seinen bestimmten Grund; es haben nämlich Versuche mich erkemnen lassen, dass der Character oder Verlauf, kurz das klinische Bild eines Geschw üres ein wesentlich anderes 
ist, wenn zur Implantation der Staphylokokken die mittleren und wenn andererseits die Rand parthien benutzt wurden. Central sitzende Staphylokokkengeschwüre haben viel mehr Neigung zur Progression, afficiren in viel höherem Grade die Iris, das Corpus ciliare, ja die vorderen $\mathrm{Ab}$ schnitte der Chorioidea, als randständige Geschwüre aus gleicher Aetiologie. Ein randständiges Staphylokokkengeschwür der Hornhaut heilt fast ausnahmslos in 3-4 Tagen ohne starke Entzündung der Iris, hie und da ohne dass es überhaupt, sicherlich nicht in bedeutendem Masse, zur Fibrinbildung, zur Eiteransammlung in der vorderen Kammer kommt. Welch andere Factoren hier noch in Betracht kommen, ob die Resorptions- resp. Diffusionsbedingungen der Proteïne und Stoffwechselproducte von der Mitte der Hornhaut aus besser sind als von der Randzone, wll ich hier nicht näher auseinander setzen, da einmal meine bezüglichen Versuche noch nicht vollstïndig abgeschlossen sind und ich ohnehin bei einer anderen Arbeit ausführlicher darauf zurückkommen muss.

Behandlung der experimentell erzeugten Geschwüre: Das eine Auge wurde immer nur mit Einträuflung von 4-8 Tropfen einer 1 procentigen Atropinlösung behandelt, das andere Auge mit derselben Quantität Atropin und subconjunctivalen Sublimatinjectionen nach den von Darier gegebenen Vorschriften. Es wurde durchschnittlich 12 bis 24 Stunden nach der Staphylokokkenimplantation, innerhalb welcher Zeit es immer zur eitrigen Keratitis mit Hypopyonbildung gekommen war, die erste Sublimatinjection vorgenommen. Die Einstichstelle befand sich ca. $3 \mathrm{~mm}$ vom Hornhautrand entfernt, eingespritzt wurde eine wechselnde Quantität 11/2 -4 Theilstriche der Pravaz'schen Spritze. Die Concentration der Sublimatlösung war meist 1:1000, in wenigen Fällen 1:2000 oder 1:800. Die Einspritzungen wurden je nach den vorhandenen Reizerscheinungen täglich oder einen Tag, hie und da auch 2 Tage überspringend, 
Experimentelle Unters. üb. 凡. Staphylokokkengeschwür etc. 59

vorgenommen. Bei 3 Versuchen habe ich gleich nach der Einimpfung der Staphylokokken in die Hornhaut die Sublimatinjection vorgenommen. - Erwähnen will ich hier kurz, dass ich auch bei vollständig normalen Augen Einspritzungen genannter Sublimatlösung unter die Bindehaut vorgenommen habe, um zu sehen, ob etwa, abgesehen ron den localen Reizerscheinungen an der Eintrittsstelle, Reiz- oder Entzündungserscheinungen im Innern des Auges auftreten würden, was absolut nicht der Fall war.

War von vornherein die Intensität der Geschwïrsbildung auf beiden Augen nicht ganz gleich, so wurden die Sublimatinjectionen auf dem Auge vorgenommen, welches in geringerem Grade afficirt erschien, um ja nicht die therapeutische Wirkung der Sublimatinjectionen zu versehleiern.

Die Zahl meiner Versuche nach dieser Richtung hin belief sich anf 40 .

Erfolge der genannten Behandlung:

A us meinen Versuchen geht mit absoluter Bestimmtheit hervor, dass den subconjunctivalen Sublimatinjectionen kein therapeutischer Nutzeffect zukommt. Der Reizzustand des Auges wird im Gegentheil dadurch meist erhöht und hält etwas länger vor. - Fine grössere Reihe von Versuchen hielt ich deshalb für nothwendig, weil bei den experimentell erzeugten Staphylokokkengeschwüren der Verlauf ein wechselnder ist, wenn auch micht so wechselnd wie der Verlauf des Ulcus corneae serpens beim Menschen. - Bemerken muss ich noch, dass ich häufig an der Einstichstelle und deren nächsten Umgebung mehr oder minder starke Verätzung der Bindehaut beobachtete.

Ich wende mich nun zur Erörterung der Frage, ob denn nicht rom bakteriologischen Standpunkt aus von vormherein ein Nutzen der subconjunctivalen Sublimatinjectionen ausgeschlossen ist. Um in dieser Hinsicht zu einem Schlusse zu kommen, wäre zunächst die Frage zu beantworten, ob in dem Hypopyon, in der 
Iris, in dem Corpus ciliare oder überhaupt im Innern des Bulbus bei dem Ulcus corneae serpens Bakterien vorhanden sind. - Während von einer Reihe von Autoren (Michel, Leber, Hess, Stromeyer, Rindfleisch, Silvestri und Andern) angegeben wird, dass sie nie oder fast nie Bakterien in dem Hypopyoneiter gefunden hätten, scheint doch noch immer die Auffassung eine sehr verbreitete zu sein, dass in dem Hypopyoneiter Bakterien vorhanden seien, dass die an ein Hornhautgeschwür sich anschliessende Entzündung der tieferen Theile des Auges auf einer Ausbreitung, Verschleppung von Mikroorganismen nach genamnten Theilen zurückzuführen sei. Ich bin nun, um endgiltig Klarheit in diese Frage zu bringen, ihrer Lösung durch eine grosse Zahl von Versuchen näher getreten. Einmal habe ich mehrere Hunderte von Schnitten durch den ganzen Bulbus oder nur den vordern. Bulbusabschnitt angefertigt und mikroskopisch untersucht, weiterhin das unter aseptischen Kautelen angesaugte Kammerwasser und den Hypopyoneiter $18 \mathrm{mal}$ bakteriologisch untersucht und zwar durch Mischen mit verschiedenen Nährböden und Anlegung von Platten. Siehenmal stammte der Hypopyoneiter von menschlichen Augen, 11 mal von Kaninchenaugen, bei denen Staphylokokkengeschwüre erzeugt waren. Der Eiter wurde 1-4 Tage nach der Staphylokokkeninfection der Hornhaut angesaugt. Die mikroskopische Untersuchung erstreckte sich auf ungefähr 60 Augen.

Auf Grund dieser ausgedehnten Untersuchungen habe ich die bestimmte Ueberzeugung, dass bei dem Staphylokokkengeschwür der Hornhaut, falls nicht bei seiner Erzeugung die Hornhaut perforirt wurde, falls es nicht selbst secundär zur Perforation der Hornhaut oder einer anderen Stelle des Bulbus geführt hat, Bakterien weder in der vorderen $\mathrm{Kammer}$, in der Iris, in dem Corpus ciliare noch sonstwo in oculo vorhanden sind. 
Experimentelle Unters. üb. d. Staphylokokkengeschwür etc, 61

Es sind demnach auch vom bakteriologischen Standpunkte aus, subconjunctivale Sublimatinjectionen absolut zu verwerfen, denn was soll das Sublimat im Auge, wenn iberhaupt keine Bakterien in demselben vorhanden sind, auf die es eventuell tödtend, schädigend einwirken könnte, es könnte demnach nur reizend, also schädlich wirken. - Nehmen wir aber nun einmal an, die 1-3 unter die Bindehaut eingespritzten Theilstriche einer Pra. vaz'schen Spritze, welche Sublimatlösung 1:1000 enthält, wïrden von den Lymphbahnen aufgenommen, wieviel könnte davon in das Auge gelangen, welche Verdünnung der Concentration würde damn noch vor sich gehen? Sicherlich eine so starke, dass etwa in der Regenbogenhaut, in dem Strahlenkörper oder sonstwo in dem Auge befindliche Mikroorganismen sich dabei wohl befinden würden.

Da bezüglich der Genese des Hypopyons immer noch nicht eine vollständige Uebereinstimmung herrscht, so war es nothwendig bei meinen Versuchen auch darauf zu achten. Besonders überzeugende Resultate lassen sich in dieser Beziehung ladurch gewinnen, dass man einmal die Fribstadien der Hypopyonbildung beobachtet, weiterhin diejenigen, wo das Hypopyon im Anschlusse an ein Hornhantgeschwür schon mehrere Tage besteht, ohne dass es zur Perforation der Hornhaut gekommen ist, wo also die Hornhaut vollgepfropft ist von Leukocyten.

Da sieht man dann im ersteren Falle, dass schon 2-3 Stunden nach der Infection der Hornhaut (in ibren mittleren Parthieen) mit Staphylokokken, eine Fibrinausscheidung auftritt, auch einzelne Leukocyten werden beobachtet, zunächst zwischen den Ciliarfortsätzen, dann auch in der vordern Kammer. Ciliarfortsätze, corpus ciliare und Iris, geraten mehr und mehr in den Zustand der Entzündung, zeigen sich durchsetzt von Leukocyten. Eine Einwanderung von Leukocyten, das Hereingelangen von Fibrin vou der Hornhaut aus ist aber so früh nach 
der Anlegung des Geschwïres absolut ausgeschlossen. Ich kann mir überhaupt nicht recht vorstellen, wie das Fibrin aus der Hornhaut in die vordere Augenkammer gelangen soll, besonders im Anfang wo das Endothel fast immer in normaler Weise vorhanden ist. Von den Endothelzellen selbst kann es doch auch nicht herrühren. In vorgeschrittenen Stadien der Geschwürsbildung fehlt das Endothel allerdings streckenweise. - Von einer Proliferation oder sonstigen Vorgängen in demselben konnte ich nichts wahrnehmen.

In späteren Stadien der Geschwürsbildung kann man nur beobachten, dass das Hornhautstroma hochgradig von Leukocyten durchsetzt ist; dass jedoch Leukocyten in einem solchen Falle durch die uuversehrte Membrana Descemetii hindurchtreten, muss ich mit aller Entschiedenheit in Abrede stellen.

Ich stehe voll und ganz auf der Seite derer, welche sagen, das Hypopyon komme zustande durch Ausscheidung einer fibrinösen Substanz und von Leukocyten aus den Gefässen der Iris und des Corpus ciliare. Allerhöchstens könnte zugegeben werden, dass ganz vereinzelte Leukocyten aus dem Randschlingennetz der Hornhaut stammend im Kammerwinkel in die vordere Kammer gelangen, doch muss ich sagen, dass mir auch dies höchst zweifelhaft erscheint. - Als ursächliches Moment für die Entzïndung, für die Reizung der Gefässwände sind die von den Bakterien der Hornhaut herrihhrenden und durch die Hornhaut hindurchdiffundirenden Proteine und Stoffwechselproducte anzusehen.

Ich habe oben dargethan, dass weder meine klinischen noch meine bakteriologischen und mikroskopischen Beobachtungen die Möglichkeit eines therapeutischen Effectes der subconjunctivalen Sublimatinfectionen bei dem Ulcus corneae serpens zulassen. Noch bleibt mir jedoch übrig, mit Bestimmtheit darzuthun, dass auch bei inficirten penetrirenden $W$ unden des Bulbus, bei septischer 
Experimentelle Unters, üb. d. Staphylokokkengeschwür etc. 63

Embolie, überhaupt überall wo in dem Auge sicher oder wahrscheinlich rorhandene Bakterien abgetödtet werden sollen, fernerhin bei den mit Bestimmtheit auf Lues zurückzuführenden Erkrankungen des Auges von einem Nutzen der subconjunctivalen Sublimatinjectionen absolut keine Rede sein kann. Um die Beweise auch hierfür zu erbringen, war es nothwendig, chemische Untersuchungen vorzunehmen.

Die chemische Untersuchung erstreckte sich sowohl auf Augen, bei denen subconjunctival auf einem oder beiden Augen Sublimateinspritzungen vorgenommen waren als auch auf solche, welche in toto in Sublimat gelegen hatten.

Die Untersuchungen wurden von Dr. med. et phil. Gürber, Assistenten für physiologische Chemie am hiesigen Physiologischen Institut in liebenswürdigster Weise vorgenommen. Den bezüglichen Bericht des Collegen Gürber lasse ich hier folgen:

"Die von Sgrosso und Scalinci behauptete Wirkung der subconjunctivalen Sublimatinjectionen setzt an sich schon als selbstverständlich voraus, dass das Sublimat von der Injectionsstelle aus sich überall hin im Auge ausbreite und so an die Orte seiner vermeintlichen Wirksamkeit gelange. Es ist deshalb nicht recht einzusehen, warum sich die beiden Forscher noch so besondere Mühe gegeben haben, das Sublimat an diesen Orten auch chemisch nachzuweisen, zumal es doch schon a priori als ganz aussichtslos erscheinen muss, von den injicirten $0,0003-0,0004 \mathrm{gr}$ Sublimat an irgend einem von der Injectionsstelle entferntern Ort etwas wiederfinden $\mathrm{zu}$ wollen, selbst wenn sich auch die ganze applicirte Menge im Bulbus verteilte und nicht schon ein grosser Teil derselben von den Formelementen der Injectionsstelle festgehalten würde, oder gar in den Kreislauf überginge.

Als geradezu unzulässig muss aber erst die Methode bezeichnet werden, nach der die beiden Forscher das Sub- 
limat im Kammerwasser, in der Cornea und dem Glaskürper u. s. w. nachgewiesen haben wollen. Sie versetzten nämlich zu diesem Zwecke das Kammerwasser mit Zinnchlorürlüsung oder legten in letztere feine Schnitte der Cornea ein und den hierbei entstandenen Niederschlag, bezw. Trübung glauben sie nach dem im mikroskopischen Bilde granschwarzem Aussehen für Calomel (?) halten zu müssen.

Wenn auch das Zinnchlorïr gewiss ein scharfes Reagens auf Quecksilberverbindungen ist, so darf es doch nicht in der oben angefuhrten Weise zur Anwendung gebracht werden, zumal die Eiweisskörper des Kammerwassers, der Coruea u. s. w. durch Zinnsalze ebenfalls gefällt werden und es ganz undenkbar ist, wie man von dem massigen Eiweissniederschlag die doch jedenfalls nur minimalen Mengen des Quecksilberniederschlages, der, wenn er wirklich Calomel $\left(\mathrm{Hg}_{2} \mathrm{Cl}_{2}\right)$ wäre, zudem nicht grauschwarz, wie Sgrosso und Scalinci meinen, sondern weiss sein müsste, unterscheiden könnte. Nun wird aber, wie in den vorliegenden Fällen, wo das Chlorzinn zweifellos im Ueberschuss angewandt wurde, das Sublimat überhaupt nicht als Calomel, sondern als metallisches Quecksilber gefällt und als solches hätten es die beiden Forscher in Form mehr oder weniger glänzender Kügelchen unter dem Mikroskope sehen müssen. Davon scheinen sie aber nichts bemerkt zu haben. Wenn wir zu all dem noch sehen, wie oftmals durch Chlorzinn schon in reinen Eiweisslösungen grauschwarze Niederschläge entstehen können, wahrscheinlich unter Bildung von Schwefelzinn, so bedarf es wohl kaum einer weiteren Begriundung, warum wir die Methode von Sgrosso und Scalinci als durchaus unbrauchbar bezeichnet haben. Es ist dies Vorgehen der beiden Forscher um so weniger zu begreifen, als es doch andere Methoden zum Nachweis von Quecksilber im thierischen Gewebe und in Flüssigkeiten giebt, die ohne mit den einfachsten analytischen Gesetzen in Widerspruch zu stehen an Empfindlichkeit 
nichts zu wünschen übrig lassen, so z. B. die Ludwig'sche Zinkamalgamprobe.

Diese Ludwig'sche Methode besteht mit einigen von uns für zweckmässig befundenen Modificationen darin, dass man das thierische Gewebe mit Salzsäure und chlorsaurem Kali aufschliesst, dann bei $100^{\circ}$ zur Trockene verdampft und so die überschüssige Salzsäure und unterchlorige Säure vertreibt. Der Rückstand wird in Wasser aufgenommen, mit einem Tropfen verdünnter Schwefelsäure die Lösung schwach angesäuert und mit reinsten Zinkspänen unter häufigem Umschütteln 24 Stunden digerirt. Hierbei bildet das Zink mit dem Quecksilber ein Amalgam. Dieses wird vielmal mit Wasser, absol. Alkohol und Aether gewaschen, in einem Vacuumschwefelsäureapparat bei $90^{\circ} \mathrm{ganz}$ wasserfrei gemacht, dann in ein scharf getrocknetes Arsenikröhrchen nach Berzelius gebracht und endlich auf $250-300^{\circ}$ erhitzt, wobei das Quecksilber sublimirt und sich im Halse des Röhrchens in Form mikroskopisch kleiner Kügelchen niederschlägt und da noch weiteren Reactionen unterworfen werden kann. Nach diesem Verfahren ist es möglich in thierischen Geweben, in der Grösse einer Cornea oder Flüssigkeiten wie Kammerwasser, noch 0,00002 gr Sublimat mit Sicherheit nachzuweisen, eine Empfindlichkeit der Methode, an die die Zinnchlorürreaktion, selbst wenn das Sublimat in wässeriger Lösung sich befände, bei weitem nicht heranreichte.

Wiewohl schon von vornherein aus den oben angeführten Gründen die Richtigkeit der von Sgrosso und Scalinci aufgestellten Behauptungen mehr als zweifelhaft erscheinen musste, haben wir doch, um ein Uebriges zu thun, einige Versuche im Sinne der genannten Forscher angestellt, indem wir 5 Kaninchen in je ein Auge bis zu $0,0005 \mathrm{gr}$ Sublimat subconjunctival applicirten und dann nach 7-30 Stunden das Kammerwasser nach der soeben beschriebenen Methode auf Quecksilber untersuchten. Aber 
in keinem der 5 Fälle war auch nur eine Spur Quecksilber zu finden. Um überhaupt die Durchlässigkeit der Augenhäute für Sublimat $\mathrm{zu}$ studiren, haben wir frisch enucleirte Bulbi 10-20 Stunden in einige cbcm Sublimatlösung von 1:4000 bis 1:1000 gelegt und dann Kammerwasser und Glaskörper auf Sublimat untersucht. Nur in den Fällen, wo die Augen mehr als 10 Stunden in den Lösungen von 1:2000 bis 1:1000 gelegen hatten, liess sich im Augeninhalt Quecksilber deutlich nachweisen. Es scheint demmach als ob Sublimat in verdünnten Lösungen nur schwer durch intacte Augenhäute hindurchginge, was auch durch folgenden Versuch bestätigt wird. Ein Bulbus wird, nachdem er 10 Stunden in einer Sublimatlösung von 1:3000 gelegen hatte, sorgfältig mit Wasser abgespült und seine Cornea auf Quecksilber untersucht, jedoch mit negativem Erfolg. Wurde dagegen vor dem Einlegen des Bulbus in die Sublimatlösung auf der Cornea ein Epithelverlust gesetzt, so ergab dieser nachher deutliche Quecksilberreaction".

Ich gehe nun über zur Besprechung einer anderen Therapie des Ulcus corneae serpens, welche vielfach, bis vor $1 / 2$ Jahr auch in hiesiger Klinik, geiibt wird, ich meine das öfters, ungetähr alle 2 Stunden, wiederholte Ausspülen des Bindehautsackes mit einer Sublimatlösung in der Concentration 1:3000 oder 1:5000. Da sich auch hier auf Grund rein klinischer Beobachtungen nur schwer ein richtiges Urtheil gewinnen lässt über den wirklichen Nutzen derartiger Ausspülungen - ein Geschwür heilt dabei oder vielleicht richtiger gesagt trotz dieser Therapie ganz gut, das andere schreitet unaufhaltsam weiter bis die ganze Hornhaut zerstört ist so betrat ich hier ebenfalls den experimentellen Weg, um eine Vorstellung über den Werth derartiger Ausspülungen zu bekommen.

Ich ging hierbei folgendermaassen vor: 
ZZunächst wurde eine normale Hornhaut continuirlich 5 Minuten lang mit Sublimat 1:3000 unter mässigem Druck irrigirt, weiterbin Hornhäute, bei denen mehr oder minder ausgedehnte und unterschiedlich tiefgreifende Defecte gesetzt waren, in gleicher Weise behandelt. Dies Letztere war deshalb nothwendig, weil ja experimentell festgestellt ist (Bellarmin off und Andere), dass eine solche Hornhaut eine grössere Durchlässigkeit für viele Substanzen besitzt. Die Augen wurden dann sofort enucleirt, die Hornhaut abgetragen und chemisch untersucht. Es ergab sich nun bei der chemischen Untersuchung folgendes Resultat:

In der normalen Hornhaut liess sich nach 5 Minuten langer Berieselung mit Sublimat 1:3000 keine Spur von Sublimat nachweisen, dahingegen drangen geringe Mengen von Sublimat in die lädirten Hornhäute ein. Zahl der Versuche im Ganzen 12. Weiterhin waren zur Feststellung der Wirkung derartiger Ausspülungen noch andere Versuche nothwendig; dieselben wurden in folgender Weise angestellt:

Es wurde zunächst wieder eine Tasche in der Hornhaut nit einer krummen Lanze gebildet, und dann in dieselbe Staphylokokken, von einer Reincultur entnommen, hineingepresst. In verschieden grossen Zeiträumen hernach wurde die Hornhaut mit einer Sublimatlösung 1:5000 und 1:3000 fünf Minuten lang berieselt, hierauf das Auge enucleirt, noch einmal mit Sublimat 1:1000 abgewaschen, dann mit steriler physiologischer Kochsalzlösung bespült, die Hornhaut unter aseptischen Cautelen abgetragen, in Stückchen zerschnitten, letztere in einen Nährboden (Agar, Gelatine) gebracht, gründlich darin umgeschüttelt und dann entsprechend lange Zeit in den Brutschrank gestellt oder bei Zimmertemperatur gehalten.

Obwohl ich 14 Versuche anstellte und die verschiedensten Zeitpunkte nach der Infection der Hornhaut wählte (11/2 Stunde bis 48 Stunden), gelang es mir nur ein 
einziges mal bei einem seit 30 Stunden bestehenden, oberflächlich gelegenen Geschwür durch 5 Minuten langes Bespülen mit Sublimatlösung 1:1000 sämmtliche Keime zu tödten. Wahrscheinlich waren hier überhaupt keine Keime mehr oder nur sehr wenige in noch relativ intactem Hornhautgewebe vorhanden; die in dem Belag des Geschwüres eventuell vorhandenen Bakterien wurden abgetötet oder mechanisch bei der Irrigation weggeschwemmt. Ausserdem weiss jeder, welcher längere Zeit bestehende Hornhautgeschwüre durch Abimpfen oder mikroskopisch untersucht hat, dass es durchaus nicht immer gelingt, Bakterien so lange Zeit nach der Anlegung des Geschwüres noch nachzuweisen.

Bei 3 weiteren Versuchen gingen nur relativ wenige Colonieen an, bei 10 jedoch wuchs eine grosse Anzahl von Colonieen.

Da nun durch das Bespülen mit einer Sublimatlösung die Bindehaut immer gereizt wird, so kann ich auf Grund vorerwähnter Versuche diese therapeutische Massnabme als nicht $z$ weckmässig bezeichnen, denn es könnte eine eventuell günstige Einwirkung nur so ihre Erklärung finden, dass ein Theil der im Bindehautsack befindlichen Mikroorganismen und deren Proteine sowie Stoffwechselproducte dadurch weggespült würden. Dies erreichen wir aber auch durch Bespülen mit einer indifferenten, nicht reizenden Flüssigkeit. Dass Einwürfe - wie „es könnte doch ein Theil der Staphylokokken getötet werden oder deren Virulenz herabgesetzt werden" absolut nicht stichhaltig sind, weiss jeder, der sich mit dieser Frage eingehend beschäftigt oder die Veröffentlichungen der letzten Jahre verfolgt hat.

Von der Ansicht ausgehend, dass der geringe antiseptische Werth des Sublimats bei diesen Versuchen besonders mit seiner eiweisscoagulirenden Eigenschaft zusammenhängt, machte ich noch Versuche mit anderen Antisepticis, welche 
keine oder nicht so starke Veränderungen der Eiweisskörper erzeugen, und zwar experimentirte ich weiter mit Hydrargyrum oxycyanatum $1 \%$ und $2 \%$, mit Jodtrichlorid 1:1000 und mit Pyoktanin (Merk'sches Fabrikat blau $2 \%$ und $1 \%$ ).

Die Versuchsanordnung war die gleiche wie bei den Berieselungen der Hornhaut mit Sublimat.

Bei 8 Versuchen mit Hydrargyrum oxycyanatum erhielt ich stets sterile Platten, bei 8 Versuchen mit Jodtrichlorid 7 mal sterile Platten, einmal Wachsthum von ungefähr 100 Colonieen, bei $3 \mathrm{Ver}$ suchen, wo ich 15 Minuten zuvor Pyoktanin $(2 \%)$ in deu Bindehautsack eingestäubt hatte, bei 1 Versuche, wo das gleiche mit Pyoktanin $(1 \%)$ der Fall war, erhielt ich stets sterile Platten.

Bei all diesen Versuchen wurde, wie ja nicht anders zu erwarten war, ein mehr oder minder starker Reiz auf die Bindehaut ausgeübt, hätte ich jedoch eines der geprüften Mittel zu empfehlen, so wäre dies das Hydrargyrum oxycyanatum.

Eine vielfach übliche Methode der Behandlung des Ulcus corneae serpens besteht fernerhin darin, dass man mittels eines schairfen Löffels oder eines sonst geeigneten Instrumentes den Geschwürsgrund und die Geschwürsränder abkratzt und so das nekrotische Gewebe entfernt. Ich habe das selbst oft gethan und es schien mix diese Maassnahme von guter Wirkung zu sein, für vereinzelte Fälle möchte ich aber eher das Gegentheil annehmen. Die Betrachtung von mikroskopischen Präparaten der verschiedensten Staphylokokkengeschwüre der Hornhaut lässt eben auch beide Möglichkeiten zu.

Liegen nämlich die Staphylokokken nur in schon nekrotischem, aufgeweichtem Gewebe, so ist es leicht denkbar, dass man durch das Abschaben einen Erfolg, vor allem eine raschere Reinigung des Geschwüres herbeiführen kann, 
ist das Gewebe aber noch relatir intact, so wird die vollständige Entfernung der Kokken nur selur viel schwerer gelingen; ferner scheint es mir besonders bei einigermaassen energischem Schaben nicht unmöglich, dass die Kokken auch in neue Saftliucken hineingepresst werden. - Eine Reihe von Versuchen, denen die bakteriologische Controle folgte, hat mich gelehrt, dass man durch kräftiges Auskratzen des Geschwüres allerdings im Stande ist, sämmtliche Staphylokokken aus der Hornhaut zu entfernen ( 8 Versuche), allein trotz alledem möchte ich das Auskratzen der Geschwüre nicht ohne Rückhalt und nur für gewisse, besonders sich eignende Fälle empfehlen. Da jedoch diese letztere Art von Fällen mir absolut nicht hinreichend klinisch charakterisirt erscheint, so muss ich das Auskratzen als einen zweischneidigen Eingriff bezeichnen. In letzterer Zeit hat Wecker das Abkratzen mit nachfolgender antiseptischer Spülung empfohlen; es ist ja gewiss nicht zu leugnen, dass damit gute Erfolge erzielt werden können, ob aber diese Maassnahme wirklich schonender ist und ebenso sicher wirkt wie die Glühhitze, möchte ich dahingestellt sein lassen. Kauterisirt man nämlich leicht den Geschwürsgrund sowie sorgtältigst die Geschwürsränder, womöglich eine Spur darüber hinaus, dann ist man absolut sicher alle Keime zerstört zu haben. 10 bezügliche Versuche sind alle in positiven Sinne ausgefallen $d$. h. die nachfolgende $A b$ tragung der Hornhaut und bakteriologische Untersuchung ergab stets sterile Platten.

Folgende Behandlung der septischen Geschwüre halte ich auf Grund meiner experimentellen und klinischen Beobachtungen für die zweckmässigste, wobei ich mir allerdings des Umstandes bewusst bin, dass es absolut nicht angängig ist, dieselben durchaus nach einer bestimmten Schablone zu behandeln, auch lasse ich die Behandlung etwaiger Complicationen speciell der so häufig, gleichzeitig 
Experimentelle Unters. üb, d. Staphylokokkengesehwür etc.

vorhandenen Thränennasencanalleiden hier ausser Betracht:

Das Auge ist für gewöhnlich unter einem Verbande zu halten. Täglich werden, falls keine Contraindication vorliegt, z. B. Neigung zur intraocularen Drucksteigerung oder die Gefahr der Perforation an einer peripheren Stelle der Hornhaut, 4-8 Tropfen Atropin (1\% Lösung) eingeträufelt. Bei dem hierzu nöthigen Verbandwechsel ist das Secret der Bindehaut zu entfernen und der Patient aufzufordern, öfters den Lidschlag wirken zu lassen, um auf diese Weise im Bindehautsack vorhandene Bakterien, Proteine, Stoffwechselproducte nach Möglichkeit nach der Nase zu abzuführen. Auf letzteren Umstand ist wohl auch die öfters meinerseits constatirte und früher von mir berichtete günstige Wirkung der Reinigung des Bindehautsackes durch Auswischen mittels Wattetupfer und gleichzeitiger Irrigation mit physiologischer Kochsalzlösung zurückzufiuhren. - Zeigt das Geschwïr Neigung zur Progression so wird kauterisirt und zwar der Grund sowohl, als die Ränder (conf. oben); allein auch wenn das Geschwiir keine ausgesprochene Neigung zur Progression zeigt, kann man kauterisiren, vielleicht weniger energisch in diesen Fällen, da es auf diese Weise möglich ist die Heilungsdauer gïnstig zu beeinflussen. Besteht ein beträchtliches Hypopyon, das keine Neigung zeigt nach gründlicher Atropinisirung zuriickzugehen, so ist eine Punction der vorderen Kammer auszuführen, um auf diese Weise Eiter und Fibringerinnsel nach Möglichkeit zu entfernen. Eventuell ist diese Maassnahme zu wiederholen. Es empfiehlt sich hierbei, die Ei11stichstelle $1-1 \frac{1}{2} \mathrm{~mm}$ vom Hornhautrande entfernt zu wählen, um Verwachsungen der Iris vorzubeugen. - Wie erklärt sich die günstige Wirkung solcher Punctionen? Ich denke mir dieselbe folgendermaassen: Durch die Entspannung der Hornhaut wird dem Vorricken der von dem Hornhautrand hereinwandernden Leukocyten Vorschub ge- 
leistet; auf die Wirkung dieser Leukocyten komme ich unten zurück. Der abnorme, ungünstig wirkende Kammerinhalt wird entfernt, gleichzeitig damit ein Theil der vorher verlegten Abflusswege der vordern Kammer wieder frei, womit die Möglichkeit einer rascheren Abführung der neu sich bildenden Entzündungsproducte und der in die vordere Kammer diffundirenden Stoffwechselproducte gegeben ist.

Ausser den genannten Maassnahmen empfiehlt es sich, öfters am Tage eine schwache Sublimat- oder Borvaselinsalbe einzustreichen oder auch das Atropin $(2 \%)$ in Salbenform zu verabreichen. Es ist hierzu am besten weisses amerikanisches Vaselin und zwar die Marke der Chesebrough Manufacturing Co. zu verwenden, da dieses Vaselin nicht reizt.

Im Verlaufe dieser, Untersuchungen drängte sich mir eine Anzahl von Gesichtspunkten auf, deren genaue Prüfung mir der Mühe werth erschien, besonders im Hinblick auf das überaus häufige Vorkommen des Ulcus corneae serpens in der ophthalmologischen Praxis. Ist es doch klar, dass die Therapie bei einem Krankheitsprocess sich aufbaut auf der Erforschung einmal der Krankheitsursache, dann der Erkenntniss der pathologisch-anatomischen Veränderungen, dass also erst nach Klarstellung dieser Punkte in therapeutischer Hinsicht wirklich zielbewusst vorgegangen werden kann und die richtigen, vernünftigen Bahnen betreten werden können.

Nun über das Ulcus comeae serpens hat sich eine ungehenere Literatur angehäuft, es liegen eine Reihe ganz hervorragender Arbeiten darüber vor und trotzdem befindet man sich noch iiber manche Punkte in der Controverse.

Was die A etiologie des Ulcus corneae serpens anlangt, so herrscht darüber ziemliche Klarheit und Einigkeit der Process. ist auf das Eindringeu von Bakterien in die Hornhaut wohl ziemlich ausnahmslos zurückzutühren; das 
Primäre ist fast regelmässig ein Substanzverlust, meist herbeigefiihrt durch ein Trauma, gleichzeitig aber oder doch bald darauf findet die Infection statt. Als solche Infectionskeime wären in erster Linie zu nennen der Staphylococcus pyogenes in seinen verschiedenen Arten; der Streptococcus pyogenes, allein auch andere Pilze z. B. Schimmelpilze können ein typisches Ulcus corneae serpens hervorbringen. Ich möchte hier kurz erwähnen, dass noch mehrere andere Namen für diese Krankheit im Gebrauche sind, meiner Ansicht nach ist am richtigsten die Bezeichnung "Ulcus corneae progrediens (serpens)" wozu dann immer noch der specielle Sitz in der Hormhaut hinzuzufügen wäre. Hierzu bemerke ich, dass das wirklich typische progrediente Hornhautgeschwür fast ausnahmslos von den mittlexen Parthieen der Hornhaut seinen Ausgangspunkt nimmt. Der Verlauf des Geschwüres ist beim Menschen, abgesehen von der Art der Infection, abhängig von individuellen Verhältnissen, so ist z. B. im Allgemeinen der Verlauf bei kräftigen jugendlichen Individuen meist ein relativ günstiger.

Nicht in gleichem Maasse haben sich die Ansichten geeinigt über das Verhalten der Bakterien in der Hornhaut und deren schliessliches Schicksal.

Während die einen sagen, die Bakterien speciell die Staphylokokken besässen nicht die Fähigkeit, sich von der Impfstelle aus, in der Hornhaut auszubreiten, bestreiten dies Andere. Ich habe nun genug der Beweise an mikroskopischen Präparaten, dass die Möglichkeit der Ausbreitung in der Hornhaut allerdings vorhanden ist, dass dies oft, ja meist der Fall ist. Warum nun dass eine Mal eine rasche Ausbreitung erfolgt, das andere Mal nicht, dies können wir wohl zur Zeit noch nicht mit absoluter Bestimmtheit sagen. In Betracht kommen hier sicher bis zu einem gewissen Grade der Infectionsmodus, die Art und die Virulenz der Infectionskeime, dann die verschiedene Widerstandstähigkeit, welche verschieden sein kann bei derselben Species, die 
sicherlich verschieden ist bei verschiedenen Species. - Für die Möglichkeit der Ausbreitung der Bakterien in der Hornhaut spricht iibrigens ja auch das klinische Bild, der progrediente Charakter mit fast absoluter Sicherheit.

Was lehrt uns nun die Betrachtung ron mikroskopischen Präparaten der verschiedensten Hornhautgeschwüre? Es unterliegt keinem Zweifel, dass die Bakterien sich in den Saftliicken der Hornhaut ausbreiten und vermehren können. In einer unterschiedlichen Zeitdauer nach dem Ein- resp. Vordringen der Kokken, auch in verschieden starkem Grade, tritt dann eine Nekrose des Gewebes um die Kokken herum eil. Dass es sich hier wirklich um eine Nekrose handelt, können wir durch Färbung z. B. nit Pikrolithioncarmin deutlich nachweisen, da bekanntlich die Pikrinsäure nekrotisches Gewebe gelb färbt. Nicht immer jedoch findet eine solche Ausbreitung in nennenswerthem Maasse oder überhaupt statt, da manchmal anscheinend sehr rasch die Nekrose rings um den Kokkenheerd eintritt und nun einen undurchdringlichen Wall bildet. Wahrscheinlich spielt in dieser Hinsicht die Virulenz der Bakterien eine Rolle, andere Einfluisse halte ich zur Zeit nicht recht für discutirbar.

Der weitere Vorgang ist dann der, dass das nekrotische Gewebe sammt den Kokken abgestossen wird. Dieser Process ist schon von verschiedenen Seiten ganz ausführlich beschrieben und brauche ich deshalb nicht weiter darauf einzugehen. Nur bezüglich der Rolle, welche die Leukocyten in dem Heilungsverlauf des Staphylokokkengeschwüres spielen, muss ich einige Bemerkungen machen. Bekanntlich ist zur Zeit noch keine Einigung darüber erzielt, ob in dem Verlauf genannter Geschwire eine Phagocytose in dem Sinne Metschnikoff's stattfindet oder ob dies nicht der Fall ist.' Erstere Ansicht wird besonders von C. Hess und Silvestri vertreten, während Andere besonders auch Leber, Michel, der Phagocytose keine nennenswerthe 
Rolle zukommen lassen, jedoch vereinzelte kokkenhaltige Leukocyten wahrgenommen haben. Ich bin num auf Grund der Durchforschung zahlreicher von Kaminchenaugen und einer Anzahl solcher von Katzenaugen stammender Präparate zu der Ueberzeugung gekommen, dass eine $\mathrm{Pha-}$ gocytose im Sinne Metschnikoff's bei dem Staphylokokkengeschwür der Hornhaut des Kaninchens und der Katze nicht stattfindet. Ich habe nie gesehen, dass Staphylokokken in Innern einer lebenden Zelle vorhanden sind; iberall da wo Kokken in einer Zelle vorhanden waren, war von einem Kerne nichts mehr zu sehen, es waren dies also keine lebensfähigen Leukocyten (Phagocyten) mehr, sondern Zellen, die schon abgestorben oder im Zerfall begriffen waren. Es würde sich nach meinen Befunden nicht darum handeln, dass Leukocyten sich der Staphylokokken bemächtigt haben, sondern um den umgekehrten Vorgang, Kokken haben sich der absterbenden Zelle bemächtigt, wahrscheinlich weil sie da einen guten Nährboden fanden. Vor allen Dingen müsste man aber anch verlangen, wenn wirklich eine Phagocytose stattfände, dass dieselbe in viel stärkerem Maasse zu Tage treten würde, man begreift dann nicht wesshalb Kokken und Leukocyten so friedlich neben einander liegen; fernerhin ergiebt sich aus der Annahme einer Phagocytose die Folgerung, dass man weit ab vom urspriinglichen Kokkenheerd kokkengefüllte Phagocyten antreffen muss. Ich habe dies nie gesehen. Um absolut sicher zu gehen, habe ich die verschiedensten Färbungen angewandt (Gram'sche Färbung und leichtes Nachfärben mit Eosin oder Congoroth, auch Alaunkarmin habe ich einigemal zur Färbung benutzt, weiterhin wurde mit Böhmer's Hämatoxylin und nach Gram, mit Pikrolithioncarmin und nach Gram getärbt). Zur weiteren Sicherheit bat ich den Privatdocenten für Bakteriologie Dr. Arens, die Befunde zu controliren und ist auch dieser der Ansicht, dass es sich 
nicht um eine Phagocytose handelt. Schliesslich erwähne ich noch, dass meine Befunde in Uebereinstimmung stehen mit den an anderen Körperstellen bezüglich der Staphylokokken festgestellten Verhältnissen, wo sich ebenfalls keine Phagocytose im Sinne Metschnikoffs ergab.

Meine Ansicht bezüglich der Wirkung der Leukocyten bei der Heilung des Staphylokokkengeschwüres geht dahin, dass besonders die von der Hormhautperipherie hereinrïckenden massenhaften Leukocyten einen Stoff ausscheiden, eine fermentative Wirkung (Leber) ausuben, wodurch dem Gewebe ein Schutz gegen weiteres Vordringen der Kokken verliehen wird. Bezüglich der weiteren Heilungsvorgänge, besonders der Abstossung des nekrotischen Gewebes, schliesse ich mich der Auffassung Lebers an.

Zum Schlusse berichte ich noch in Kürze über Versuche mit Stoffwechselproducten and Proteïnen des Staphylococcus pyogenes aureus. Die Stoffwechselproducte und Proteïne erhielt ich auf folgende Weise:

Nährbouillon wurde mit einer Staphylokokkenreinkultur inficirt und 6-30 Stunden lang in den Brutschrank gesetzt. Die Bouillon wurde dann durch einen Thoncylinder mittelst Aspiration durch die Wasserluftpumpe filtrirt; zuvor jedoch wurde stets eine Controlplatte angelegt, um zu sehen, ob auch nicht eine Verunreinigung der Bouillon inzwischen oder bei der Impfung eingetreten war. Das Gleiche war der Fall von dem Filtrate, um zu sehen, ob dasselbe wirklich steril war. Das Aussehen des Filtrats war ein leicht tribes, dunkel bräunliches. - Wässerige Aufschwemmungen zu benutzen hielt ich nicht für zweckmässig, da ich aus frïheren Untersuchungen weiss, dass die Staphylokokken durch Wasser in ihren Lebensbedingungen beeinträchtigt werden können und ausserdem die Bildung neuer Stoffwechselproducte im Wasser unmöglich ist. Dampfte ich das Filtrat auf einen geringen Theil seines Volumens ein, so wurde die gleich $z u$ besprechende Wirkung desselben verstärkt. 
Die Versuchsanordnung war folgende:

In den Bindehautsack wurden 1-3 Stunden lang in Pausen von 5-10 Minuten kleine Mengen der gewonnenen Stoffwechselproducte und Proteine gebracht. Es gelang auf diese Weise bei unverletzter Hornhaut, viel leichter und stärker jedoch bei einer Hornhaut, an welcher Epithelverluste erzeugt waren, Hyperämie, Schwellung der Conjunctiva sowie fibrinös-eitrige Entzündung der Regenbogenhaut und des Strahlenkörpers zu erzeugen.

Nahezu die gleichen Erscheinungen liessen sich auch dadurch hervorrufen, dass ich statt der Stoffwechselproducte und Proteine von einer Reinkultur des Staphylococcus pyogenes aureus immer wieder zahlreiche Keime in den Bindehautsack hereinbrachte. - Wurden die Stoffwechselproducte und Proteine in die vordere Kammer oder den Glaskörper injicirt, so kam es zu einer heftigen Entzündung, es trat eine starke Erweichung, gallertartige Beschaffenheit des Bulbus ein und meist Perforation an der Corneoskleralgrenze.

Saugt man das Kammerwasser und Hypopyon bei einem Ulcus corneae progrediens mit der Pravaz'schen Spritze an und injicirt es in die Homhaut, die vordere Kammer oder in den Glaskörper, so wird überhaupt keine oder eine kaum nennenswerthe, rasch vorïbergehende Entzïndung hervorgerufen.

Von weiteren Versuchen mit Stoffwechselproducten will ich nicht berichten im Hinblick auf die vorliegenden Untersuchungen (Leber, de Christmas).

Mir kam es bei diesen Versuchen nur darauf an, die Ueberzeugung zu gewinnen, dass die Entzündung der Iris und des Corpus ciliare bei den Hornhautgeschwüren, sowie bei sehr heftigen Bindehauterkrankungen auf einer Fernwirkung der Bakterien beruht 
und hervorgerufen wird durch dereu Stoffwechselproducte und Proteine. Dieser Beweis dürfte erbracht sein.

Da in verschiedenen Veröffentlichungen der letzten Jahre, besonders in der Arbeit Lebers „Ueber die Entstehung der Entziindung und die Wirkung der entzündungserregenden Schädlichkeiten", sowie in der Abhandlung von Silvestri „Experimentelle Untersuchungen über septische Keratitis", die Literatur eingehend berücksichtigt wurde, ging ich darauf in vorliegender Arbeit nur soweit ein, als es unbedingt nothwendig war, doch finden sich, wie ich hoffe, fast alle bezüglichen Abhandlungen in dem beigegebenen Literaturverzeichnisse.

\section{Literatur.}

1. Alt, Zur Lehre vom Hornhantabscesse. v. Graefe's Archiv XVI. 1. 1870.

2. Bach, L., Ueber den Keimgehalt des Bindehautsackes, dessen künstliche und natürliche Beeinflussung, sowie über den antiseptischen Werth der Augensalben. v. Graefes Archiv XL. 3. 1894.

3. Bötteher, Experimentelle Untersuchungen über die Entstehung der Eiterkörperchen bei der traumatischen Keratitis. Vireh. Arehiv. Bd. LVIII, Heft 3. 1873.

4. - Ueber die circumseripte Keratitis. Vireh. Archiv, Bd. LXII. Heft 4. 1874.

5. Buchner, Die chemische Reizbarkeit der Leukocyten und deren Beziehung zur Entzündung und Eiterung. Berl. klin. Wochenseh. No. 47. 1890,

6. Brieger, Weitere Untersuchungen über Ptomaine. Berlin 1885 und 1886. 
Experimentelle Unters. ül. d. Staphylokokkengeschwür ete. 79

7. Bellarmin off, L., Untersuchungen mit den quantitativen calorimetrischen Methoren über die Resorption in die vordere Kammer. ₹. Graefe's Archiv XXXIV. 3. S. 38.

8. Colzi, Sulla etiologia della ostemielite acuta. Lo Sperimentale TLXIV. Page 11, 12. 1890.

9. Cohnheim, Die traumatische Keratitis. Berlin 1873. -Noch einmal die Keratitis. Virch. Archiv. Bd. LXI. Heft 3. 1874.

10. J. de Christmas, Recherches experimentales sur la suppuration. Ann. de l'institut Pasteur. 1888. T. II. p. $469-478$.

11. Darier, A., Des injections sous-conjonetivales de sublimé. Annal. d'Oculist. TOIX. p. 241.

12. - Behandlung u. Prophylaxe der infectiösen Processe nach Staroperationen. Bericht über die 23. Vers. d. ophth. Gesellschaft z. Heidelberg. S. 99.

13. - Des injections sous-conjonctivales de sublimé dans les maladies de la choroide et de la rétine. Extrait du Compt. rend. de la société françáse d'Ophth. Séance du 4. mai.

14. - Un nouveau traitement de la chorioidite centrale et de la chorioidite disséminée. Verhandl. đ. X. internat. med. Congresses z. Berlin. IV. 2. S. 91.

15. - Des injections sous-conjonctivales de sublimé en thérapeutique oculaire, Arch. d'Ophth. p. 449.

16. Decanx, De l'origine microbienne des kératites et de leur traitement. Thèse de Paris. J.-B. f. Ophth. 1890, p, 190.

17. Dolschenkow, W., Impfung faulender Substanzen auf Kaninchenhornhant. Centralbl. für die mediz. Wissensch. 1873. S. 657 u. 673 .

18. Eberth, Experimentelle Untersuchungen über die Entzündung der Hornhaut. Untersuch. aus dem pathol. Institut zu Zürich. Heft 2. 1874.

19. - Die centrale Keratitis. Ibil. Heft 3. 1875.

20. - Diphtheritis der Cornea. Comespondenzblatt der Schweizer Aerzite. 1873.

21. Fortunati, A., Antisepsi nelle suppurazione sperimentali della cornea. Spezimentale Firenze p. 23 . J.-B. f. Ophth. 1889.

22. - Azione degli stafilococchi piogeni nelle ferite della cornea. Bolletins d' Oculistica. No. 16-17. 1888. 
23. Frisch, Experimentelle Studien uber die Verbreitung der Fäulniss-Organismen in den Geweben $u$, die durch Impfung in die Cornea mit pilzhaltigen Flüssigkeiten hervorgerufenen Entzündungserseheinungen. Erlangen 1874.

24. Fuchs, Lehrbuch der Augenheilkunde.

25. Gallengo, Camillo, Contribuzioni allo studio della cheratiti superficiali infettive. Giom. della R. Acad. die Medicine di Turino. No. 34.

26. - Alcune ricerche sperimentale sul trattamento della tuberculosi primitive della cornea e dell' mede. Parma. Novembre 1891.

27. Gagarine, V., Subconjunctivale Sublimatinjectionen. Thèse de Doctorat. St. Petersburg 1873. (Russisch.)

28. Gallemaerts, Du traitement des affections oculaires par les pales injections sous-conjonctivales. Bull. Acad. rog. de méd. Belg. Bruse. VII. p. 490.

29. Hess, C., Weitere Untersuehungen zur Phagocytenlehre. Virch. Arch. Bd. CX. Heft 2. 1887.

30. - Ueber das Staphylokokkengeschwür der Hornhaut. Bericht der XIX. Vers. d. ophth. Gesellschaft zu Heideloerg. S. 37. Discussion S. 38.

31. F. A. Hoffmann u. v. Recklinghausen, Ueber die Herkunft der Eiterkörperchen. Centralbl. f. d. med. Wissensch. 1867. No. 31.

32. F. A. Hoffmann, Ueber Eiterbildung in der Cornea. Virch. Arch. XLII. 1868.

33. - Ueber Keratitis. Die Entstehung des Hypopyon. Sitzungsb. der XVII. Vers. der ophth. Gesellsch. z. Heidelberg. 1885 . S. 70 .

34. F. Horner, Keratitis mycotiea. Ber. d. ophth. Ges. 1875. Zehend. M. Bl. XIII. S. $442-445$.

35. Axel Rey und C. Wallis. Experim. Untersuchungen über die Entzündung der Hornhaut. Virch. Arch. Bd. LV. Heft 3. 1873.

36. Leber, Die Entstehung der Entzündung u. die Wirkung der entzündungerregenden Schädlichkeiten. Leipzig 1891.

37. Limbeck, Klinisches und Experimentelles über entzündliche Leukocyten. Zeitschr. f. Heilkunde B. X. 1890.

38. Metschnik off, Ueber Phagoeytose. Virch. Arch. Bd.108.109.

39. Lübbert, Biologische Spaltpilzuntersuchung. 1886.

40. Ortmann, Experimentelle Untersuchungen über centrale Keratitis. Inaug:-Diss. Königsberg. 1884. 
Experimentelle Unters, äb. d. Staphylokokkengeschwür etc. 81

41. Petruschky, Untersuchngen ïber die Immunität des Frosehes gegen Milzbrand. Beitr. zur pathol. Anatomie u. zur allgem. Pathologie v. Ziegler u. Nawwerk. 13d. III. 1888.

42. Michel, Lehrbuch der Augenheilkunde. 2. Aufl. Wiesbaden.

43. G. Rindfleisch, Michel, Ueber septische Impf-Keratitis. Verh. d. physik. Gesellsch. z. Würzb. Bd. XXII. 1888.

44. Ribbert, Deutsche medic. Wochenschrift 1884.

45. - Die pathol. Anatomie u. die Heilung der durch den Staphylococcus pyogenes aureus hervorgerufenen Erkrankungen. Bonn, Fr. Cohen. J.-B. f. Ophth. 92. p. 194.

46. Perinoff (Peounow), Subconjunctivale Sublimatinjectionen in der ophth. Praxis. Wiestnik Ophthalm. Juli--Oetober 1895.

47. Sattler, Ueber die in Thränensackeiter enthaltenen Infectionskeime und ihr Verhalten gegen Antiseptika. Bericht der Heidelb. ophth. Gesellsch. 1885. S. 18.

48. - Die Bedeutung der Bakteriologie für die Augenheilkunde. VII. period. ophth. Congress. Heidelb. 1888. S. 377.

49. Schmidt-Rimpler, Lehrbuch der Augenheilkunte.

50. Sgrosso, P. e Noé Scalinei, Le iniezioni sottconjuntivali e intertenoniane di sublimato corrossivo nelle cura di alcune affezioni oculari. Ricerche cliniche e sperimentali. Rendicontro dell XIII. Congresso della Associazione Oftalmologica Italiano. Riunione di Palermo, April 1892. Annali di Oftalmologia anno XXII. S. 509.

51. Silvestri, A., Experimentelle Untersuchungen über sept. Keratitis. v. Graefe's Arch. XXXVII. Bd. 2. Abth. S. 220.

52. Steinhaus, Die Aetiologie der acuten Eiterungen. Deutsche med. Wochensch. 1883. No. 32.

53. Stricker, Studien etc. 1870 . S. 1 u. ff. u. Arch. f. Augenund Ohrenheilkunde. V. 1.

54. Stromeyer, Ueber die Ursache der Hypopyon-Keratitis. Archiv f. Ophth. XIX. 2. \$. 1. 1874.

55. Schweinitz, de, Subconjunctival injection of corrossive sublinate. Therapeutic Gazette 15. jun. (cf. Revue générale d' Ophth., kein Referat.)

56. Wolfheim, Ein weiterer Beitrag zur Phagocytenlehre. Beiträge z. pathol. Anatomie u. allgem. Pathologie von Ziegler u. Nauwerk. T. V-15. S. 405 .

57. Verdese, Recherches expérimentales sur la perforation précoce de la membrane de Descemete dans les processes ulcératitis infectieuses de la cornée. Archives d'ophth. 'T.IX. 1889. S. 147. 
58. Belohous, Nature et traitement des ulères de la comée à hypopyon.' Thèse de Paris. 1893.

59. Borlée, Reflexion sur l' emploi des injections sous-conjunctivales de sublimé dans les affections oculaires, du traitement des conjunctivités purulentes, des inflammations de l'iris et de la chorioide. Bull. Acad. roy. de méd. de Belg. Brux. VII. p. 596.

60. Bribosia, fils., Injections sous-conjunctivales de sublimé en thérapeutique oculaire. Journ. de la policlinique de Nancy. Déc. 1893.

61. de Wecker, Traitement des ulcères et des abscès exulcérés de la cornée par le raglage et l'inrigation. Annales d'Oculistique TCX. 1. Livraison. Juli 1893.

6ะ. Gepner, jr. B. R., Ueber subeonjunetivale Sublimateinspritzungen. Hirschberg, Centralbl. f. Augenheilkunde. Jan. 1894.

\section{Nachtrag.}

Gelegentlich eines kurzen Vortrages über obiges Thema auf dem internationalen Congress zu Edinburg wurden mir theils in der Discussion, theils privatim einige Finwürfe über meine Versuche mit subconjunctivalen Sublimatinjectionen gemacht, auf die ich hier mit wenigen Worten eingehen möchte, nachdem ich darüber auch mit Dr. Gürber Rücksprache genommen habe.

Zunächst der Einwurf: es sei eventuell möglich, dass an der Injectionsstelle ein Depot von Sublimat geschaffen wuirde, von wo aus kleinste Mengen des Sublimates längere Zeit hindurch zu den benachbarten Gegenden des Auges, speciell zur Hornhaut gelangen könnten, und in dieser langdauernden Einwirkung wenn auch kleinster Mengen sci vielleicht die Erklürung für die behauptete günstige Wirkung der subconj. Subl. inj. zu finden.

Ich möchte dazu Folgendes bemerken: Ich glaube, dass vom Bindegewehe, dessen Zellen, vielleicht auch von den elastischen Fasern, welche oben von Dr. Gürber als Formelemente bezeichnet wurden, Sublimat gebunden werden 
kann, werden doch die genannten Gebilde durch Sublimat gehärtet, d. h. ihr Eiweiss oder ihre eiweissartigen Bestandtheile durch dasselbe ausgefällt. Die Menge des so zurückgebliebenen Sublimats dürfte aber nur verschwindend gering sein, da der grösste Theil des injicirten Sublimates doch von den Eiweisskörpern der Gewebsflüssigkeit occupirt werden muss. Zwar wird es in diesem Falle kaum zu einer Fiweissfällung kommen, da die Anwesenheit von Kochsalz, auch von Harnstoff, eine solche nicht zu Stande kommen lässt. Es ist nun nur fraglich, ob das so immer noch circulirende Sublimat nicht doch noch durch Formelemente festgehalten wird. Dass von Letzteren das Sublimat aber allmählich wieder losgelassen wird, unterliegt keinem $Z_{w}$ eifel; dagegen dürfte es nach aller Erfahrung über die Ausscheidung des Quecksilbers aus dem Organismus sicher sein, dass dieses Loslassen nur sehr langsam vor sich geht. Sicheren Aufschluss über den gemachten Einwurf könnte nur folgendes Experiment geben: Nach subconjunctivaler Injection von Sublimat lässt man 3-4 Tage verstreichen und untersucht dann die Injectionsstelle auf Sublimat. Wie dies Experiment aber auch ausfallen sollte, so halte ich doch ein solches Sublimatdepot für den therapentischen Erfolg für bedeutungslos, weil die Mengen, um die es sich hier handelt, nur unendlich klein sein können.

Ferner wurde geltend gemacht, dass man doch das Thierexperiment nicht ohne weiteres auf den Menschen übertragen dürfe.

Das ist zweifellos; die Nichtbeachtung dieses Grundsatzes hat schon zu den unangenehmsten Irrthümern geführt und besonders mag das gelten für das Gebiet der experimentellen Pathologie und Therapie. Leider giebt es aber keine bestimmten Anhaltspunkte, nach denen man das Thierexperiment übertragen könnte.

Nun weiss ich aus meinen Versuchen und es ist dies ja auch sonst bekannt geworden, dass das Kaninchen viel 
84 Ludwig Bach. Experinentelle Untersuchungen etc.

refractärer sich verhält gegenüber den Staphylokokken und Streptokokken als der Mensch, es liessen sich daher therapeutische Erfolge, sofern sie günstig ausfielen, nicht von dem Kaninchenauge auf das Menschenauge uibertragen. Wenn aber wie hier der Erfolg einer Therapie sich am Kaninchenauge ungünstiger gestaltet als es vom Menschen behauptet wird, dann dürfte das Experiment am Kaninchen doch zu den stärksten Zweifeln an der Richtigkeit der von uns angefochtenen Behauptungen berechtigen.

Will man nun aus dem einen oder anderen Grunde bei der therapeutischen Maassnahme der subconjunctivalen Injectionen bleiben, so dürfte sich dazu eine Anzahl anderer Antiseptika viel besser eignen als das Sublimat. 